05

\title{
Исследование оптического Мо/Мо нанокристаллического покрытия, полученного напылением с одновременным ионным распылением
}

\author{
(c) А.В. Рогов, ${ }^{1,2}$ Ю.В. Мартыненко, ${ }^{1,3}$ Ю.В. Капустин, ${ }^{1,2}$ С.С. Фанченко, ${ }^{1}$ Н.Е. Белова ${ }^{1}$ \\ ${ }^{1}$ Национальный исследовательский центр „Курчатовский институт“, \\ 123182 Москва, Россия \\ ${ }^{2}$ Координационный центр „Управляемый термоядерный синтез - международные проекты“, \\ 123182 Москва, Россия \\ ${ }^{3}$ Национальный исследовательский ядерный университет «МИФИ», \\ 115409 Москва, Россия \\ e-mail: alex-rogov@yandex.ru
}

(Поступило в Редакцию 14 марта 2017 г. В окончательной редакции 3 ноября 2017 г.)

\begin{abstract}
Представлены результаты исследования структуры, оптических свойств и стойкости к распылению отражающего Мо-покрытия, полученного методом магнетронного напылении с одновременным низкоэнергетическим ионным распылением в режиме превышения скорости напыления над скоростью травления. В качестве подложки использовалось Мо-поликристаллическое зеркало. Показано, что покрытие, сформированное в таких условиях, имеет текстурированную нанокристаллическую структуру с малым разбросом размеров кристаллитов и обладает высокой устойчивостью к распылению. Выявлено, что спектральный коэффициент отражения такого Мо-покрытия отличается от спектрального коэффициента отражения поликристаллического и монокристаллического Мо, а наблюдаемое отличие обусловлено влиянием структуры покрытия на его оптические свойства. Предложена теоретическая модель процесса формирования покрытия.
\end{abstract}

DOI: 10.21883/JTF.2018.05.45901.2243

\section{Введение}

Одним из основных параметров при выборе материала первого зеркала для оптических диагностик международного термоядерного реактора ИТЭР является стойкость к распылению, т.е. сохранение оптического качества при равномерном распылении отражающей поверхности. Также требуется обеспечить максимальный коэффициент отражения в рабочем диапазоне длин волн оптического излучения для уменьшения габаритов зеркала и его высокую стойкость к тепловым нагрузкам и распылению атомами перезарядки. Этим требованиям отвечают зеркала, изготовленные из тугоплавких металлов, наиболее перспективными из которых являются $\mathrm{Rh}$, Мо и W [1].

Зерна в поликристаллическом металле имеют произвольную кристаллографическую ориентацию. В то же время известно, что коэффициент распыления монокристаллов зависит от их кристаллографической ориентации [2], поэтому даже незначительное распыление отражающей поверхности поликристаллических зеркал приводит к формированию рельефа с характерным размером, равным размеру зерен $(1-100 \mu \mathrm{m})$. За счет этого происходит резкое ухудшение оптических свойств зеркала. По этой причине поликристаллические зеркала не используются при наличии существенного распыления.

Наилучшими показателями по устойчивости к распылению обладают зеркала, изготовленные из монокристаллов [3]. Для разрабатываемой в России диагностики „Спектроскопия водородных линий“ (СВЛ) в качестве материала для изготовления зеркала, принимающего из- лучение из плазмы (первого зеркала), используется монокристаллический Мо, обладающий высокой устойчивостью к распылению и приемлемым коэффициентом отражения $(R=55-60 \%)$ в рабочем диапазоне длин волн регистрируемого излучения $(\lambda=450-700 \mathrm{~nm})$. Размер опытного образца Мо-монокристаллического зеркала (НПО „Луч“, г. Подольск) для этой диагностики составляет $200 \times 50 \mathrm{~mm}$ [4]. В настоящее время максимальный диаметр выращенных Мо-монокристаллов не превышает $150 \mathrm{~mm}$, поэтому зеркало было изготовлено из двух полированных монокристаллических Мопластин, закрепленных на Мо-поликристаллической подложке методом диффузионной сварки [5]. Такие зеркала уникальны и отличаются крайне высокой стоимостью.

Альтернативой могут стать металлические зеркала, изготовленные из специальных многокомпонентных аморфных сплавов, исследовавшихся в [6]. Применение таких материалов в условиях ИТЭР не регламентировано и требует дополнительных исследований: в процессе эксплуатации могут возникнуть эффекты радиационной сегрегации компонентов сплава и рекристаллизации при нагреве, что неизбежно повлияет на устойчивость зеркала к распылению.

Высокой устойчивостью к распылению обладают Мозеркала со столбчатым нанокристаллитным отражающим покрытием $[7,8]$ на поликристаллических подложках. Поскольку зеркала данного типа изготавливаются методом вакуумного напыления, отсутствуют технологические ограничения для размера зеркал. Однако столбчатая структура покрытий данного типа на несколько порядков увеличивает коэффициент диффузии дейтерия 
через покрытие, что может привести к его разрушению за счет эффекта ионно-стимулированного диффузионного блистеринга [9], который может возникнуть, например, при использовании плазменной системы очистки зеркал, встроенной в узел первого зеркала оптических диагностик ИТЭР [10].

Другим типом устойчивых к распылению покрытий могут стать однокомпонентные нанокристаллические покрытия. В соответствии с классификацией Р. Зигеля [11] такие материалы относятся к объемным однокомпонентным наноструктурным материалам. При размере кристаллитов $\sim 10 \mathrm{~nm}$ негативный эффект от различия в скорости их распыления будет незначительным за счет малости размеров формируемого при этом рельефа по отношению к длине волны передаваемого зеркалом излучения. Основные требования к таким покрытиям - их однородность и малая дисперсия размеров кристаллитов. В настоящей работе исследовались структура и оптические свойства Мо-покрытия данного типа, полученного оригинальным методом с использованием магнетронного напыления с одновременным распылением формируемого покрытия низкоэнергетическими ионами $\mathrm{Ar}^{+}$ в разряде с полым катодом, когда скорость осаждения выше скорости травления.

\section{Оборудование и методика нанесения покрытия}

В качестве подложки применялось Мо-полированное поликристаллическое зеркало в виде диска с размерами $\varnothing 25 \times 3 \mathrm{~mm}$ (изготовитель - НПО „Луч“, г. Подольск). Материал зеркала: молибден чистый вакуумной плавки (МЧВП).

Для улучшения адгезионных свойств покрытия непосредственно перед напылением проводилась финишная очистка подложек от остаточных загрязнений в сеточном цилиндрическом полом катоде [12]. Размеры полого катода: $\varnothing 65 \times 140 \mathrm{~mm}$. Он был изготовлен из нержавеющей сетки с ячейкой $2.5 \times 2.5 \mathrm{~mm}$ и толщиной $\approx 0.5 \mathrm{~mm}$, покрытой Мо. Этим обеспечивались химическая однородность покрытия при наличии частичного переосаждения продуктов эрозии и увеличение эффективности распыления. Подложка размещалась внутри полого катода на его донышке - подложкодержателе. При очистке подложек в качестве анодного электрода использовался малоразмерный планарный магнетрон с дисковым катодом и встроенным изолированным кольцевым анодом. Он размещался со стороны открытого конца полого катода соосно с ним. Магнитная система магнетрона состояла из $\mathrm{NdFeB}$-постоянных магнитов с размерами: периферийный кольцевой магнит - $18 \times \varnothing 25 \times 5 \mathrm{~mm}$, центральный цилиндрический магнит - $\varnothing 10 \times 10 \mathrm{~mm}$. Размер Мо-катода магнетрона $\varnothing 25 \times 3 \mathrm{~mm}$. В качестве рабочего газа использовался $\mathrm{Ar}$. Как было показано в $[13,14]$, при таком инверсном

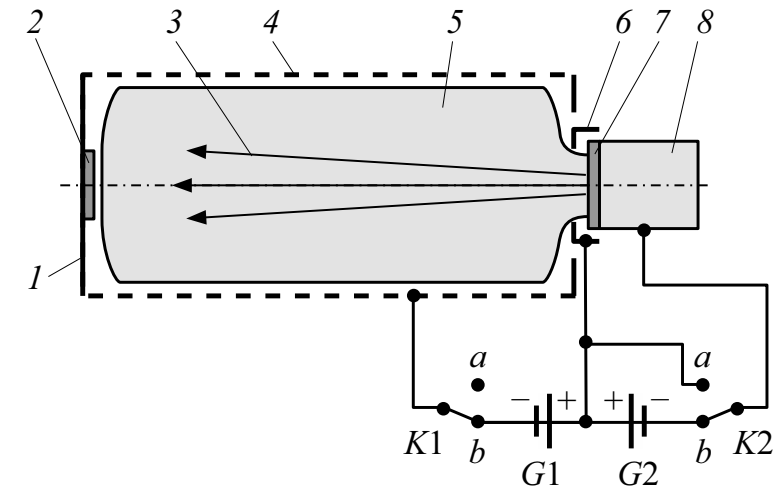

Рис. 1. Схема эксперимента: 1 - подложкодержатель, 2 - подложка, 3 - поток распыленных в магнетроне атомов, 4 - цилиндрический сеточный полый катод, 5 - комбинированный разряд магнетрон - полый катод, 6 - изолированный анод магнетрона, 7 - распыляемый катод магнетрона, 8 - корпус магнетрона, $G 1$ - источник питания полого катода, $G 2$ - источник питания магнетрона, $K 1$ и $K 2-$ переключатели.

включении магнетрон функционирует в качестве своеобразного ионного источника. При этом диапазон рабочих давлений газового разряда в режиме полого катода сдвигается в область более низких давлений. Минимальное давление в этом режиме составило $\approx 4 \cdot 10^{-4}$ Torr. Минимальное рабочее давление для использованного магнетрона в режиме напыления составило $\approx 2 \cdot 10^{-3}$ Torr. Это обеспечило возможность быстрого перехода от очистки к напылению. В результате были получены высокие адгезионные свойства покрытия без дополнительного нагрева подложки (высокая адгезия подтверждается отсутствием отслаивания на разломе зеркала с покрытием, см. далее). Финишная очистка подложки перед напылением проводилась при $P_{\mathrm{Ar}} \approx 2 \cdot 10^{-2}$ Torr, $U_{h c}=400 \mathrm{~V}, I_{h c}=100 \mathrm{~mA}, T=2 \mathrm{~min}\left(U_{h c}\right.$ и $I_{h c}-$ напряжение и ток разряда с полым катодом). Толщина распыленного слоя составила $h \approx 15 \mathrm{~nm}$.

Этот же магнетрон использовался для напыления покрытия с одновременным частичным распылением осаждаемого на подложку материала (напыление, ассистированное ионным распылением). В этом режиме одновременно включался разряд в полом катоде, обеспечивающий распыление, и магнетронный разряд для напыления покрытия. Изолированный анод магнетрона использовался в качестве анодного электрода одновременно для магнетронного разряда и разряда с полым катодом. Схема эксперимента представлена на рис. 1.

Для питания разрядов применялись два регулируемых источника питания постоянного тока, обеспечивающих стабилизацию напряжения, тока или мощности разряда. Максимальное выходное напряжение и мощность каждого их них составляли $720 \mathrm{~V}$ и $300 \mathrm{~W}$ соответственно. Переход от режима очистки к режиму напыления покрытия осуществлялся без изменения рабочего давления посредством коммутации источников питания $G 1$ 
и $G 2$ переключателями $K 1$ и $K 2$. Очистка подложек проводилась, когда $K 1$ находился в положении $(b)$, а $K 2$ в положении $(a)$. Напыление $-K 1$ в положении $(b)$, $K 2$ в положении $(b)$. При таком совместном включении магнетрона и полого катода между этими разрядами происходит обмен заряженными частицами, что обеспечивает их взаимное влияние: формируется комбинированный газовый разряд. Напыление покрытия проводилось при давлении $P_{\mathrm{Ar}}=1 \cdot 10^{-2}$ Torr. Напряжение и ток разряда в полом катоде $U_{h c}=420 \mathrm{~V}, I_{h c}=60 \mathrm{~mA}$, а магнетронного разряда $U_{m}=300 \mathrm{~V}, I_{m}=165 \mathrm{~mA}$. Температура подложки в процессе нанесения покрытия не превышала $150^{\circ} \mathrm{C}$. Длительность процесса напыления $T=120 \mathrm{~min}$. При этом толщина полученного покрытия $H \approx 1 \mu \mathrm{m}$. Оценка толщины распыленного или напыленного слоя проводилась методом взвешивания с использованием аналитических весов CAS CAUW 220D с точностью $0.01 \mathrm{mg}$ (при расчете толщины использовалось табличное значение плотности для поликристаллического молибдена $\rho_{\text {Мо }}=10.2 \mathrm{mg} / \mathrm{mm}^{3}$ ).

\section{Результаты экспериментальных исследований}

\section{Рентгеноструктурные исследования}

Рентгенодифракционные исследования полученной молибденовой пленки проводились на дифрактометре Bruker D8 - Discover в геометрии Зеемана-Болина. В этой схеме источник, образец и детектор находятся на фокусирующей окружности, и в отражении участвуют дифракционные плоскости, составляющие с поверхностью угол, равный $\left(\theta_{\mathrm{B}}-\alpha\right)$, где $\theta_{\mathrm{B}}-$ угол Брэгга, $\alpha-$ малый фиксированный угол падения. Необходимость использования этой схемы связана с минимизацией вклада в дифракцию отражения от подложки и увеличением светосилы эксперимента, так как за счет малого угла падения эффективная толщина пленки увеличивается в десятки раз. Угол выхода зондирующего излучения при этом равен $\left(2 \theta_{\mathrm{B}}-\alpha\right)$. Покрытие исследовалось при $\alpha=2^{\circ}$. Глубина проникновения рентгеновских квантов с энергией $8.05 \mathrm{keV}(\mathrm{Cu}, \lambda=0.154 \mathrm{~nm})$ составила $0.22 \mu \mathrm{m}$, что в несколько раз меньше толщины исследуемого слоя $H$. На рис. 2 представлена нормализованная дифрактограмма покрытия с учетом влияния больших углов дифракции на измеряемую интенсивность

$$
I_{\text {norm }}=I_{\exp } \sin ^{2}\left(2 \theta_{\mathrm{B}}-\alpha\right),
$$

где $I_{\exp }-$ экспериментально измеренное значение интенсивности.

В данной геометрии не могут наблюдаться отражения от плоскостей, параллельных поверхности пленки, и отсутствие на дифрактограмме пика отражения (200) свидетельствует о том, что пленка текстурированная и есть выделенные ориентации, например $\langle<001\rangle$. Для использованной измерительной схемы наблюдаемые кратные

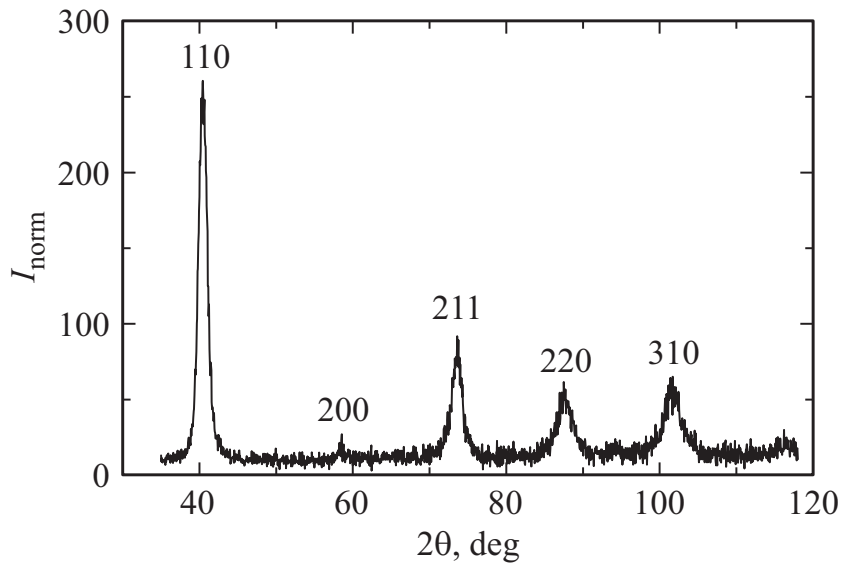

Рис. 2. Нормализованная дифрактограмма Мо-нанокристаллического покрытия $(\lambda=0.154 \mathrm{~nm})$.

Таблица 1. Экспериментальные и расчетные данные для наблюдаемых в эксперименте отражений

\begin{tabular}{c|c|c|c|c}
\hline $\begin{array}{c}\text { Отражение } \\
(h k l)\end{array}$ & $\begin{array}{c}\theta_{\mathrm{B}}^{h k l}, \\
\operatorname{deg}\end{array}$ & $\begin{array}{c}D_{\min }^{h k l}, \\
\mathrm{~nm}\end{array}$ & $\begin{array}{c}\text { Нормаль } \\
\text { текстуры }\end{array}$ & $\begin{array}{c}\text { Угол между плоскостью } \\
\text { текстуры и плоскостью } \\
\text { отражения, deg }\end{array}$ \\
\hline 110 & 20.3 & 6.4 & $\langle 221\rangle$ & 19.5 \\
211 & 36.8 & 4.6 & $\langle 221\rangle$ & 35.3 \\
220 & 43.8 & 3.6 & $\langle 221\rangle,\langle 001\rangle$ & 45 \\
310 & 50.9 & 4.0 & $\langle 110\rangle$ & 47.9 \\
\hline
\end{tabular}

отражения относятся к разным семействам плоскостей, находящихся под углом друг к другу, следовательно, отражение (220) не является кратным к наблюдаемому отражению (110), поэтому при определении размеров областей когерентного рассеяния определяется лишь наименьшее значение для каждого направления без учета вклада деформационного напряжения.

В табл. 1 приведены данные, полученные для наблюдаемых в эксперименте отражений: значения брегговских углов $\theta_{\mathrm{B}}^{h k l}$, среднее наименьшее значение областей когерентного рассеяния в направлении вектора обратной решетки $\left\langle D_{\min }^{h k l}\right\rangle$, определенное при обработке данных по формуле Шеррера, а также направления компонентов текстуры, при которых эти отражения могут наблюдаться, и углы между нормалью к плоскости отражения и направлениями компонентов текстуры.

Наличие пика (310) свидетельствует о наличии текстуры $\{110\}$. Присутствие на дифрактограмме в данной геометрии отражений (110) и (211) связано с выделенным направлением $\langle 221\rangle$, так как углы между нормалями к данным плоскостям и нормалью к плоскостям $\{221\}$ близки к значению $\left(\theta_{\mathrm{B}}-\alpha\right)$. Наличие отражения $(220)$ на дифрактограмме может быть связано как с текстурой $\langle 221\rangle$, так и с направлением $\langle 001\rangle$. УГловое отклонение с данными направлениями примерно $3^{\circ}$ и находится в пределах разумной разориентации для текстурной 
компоненты. Для отражения (200) расхождение с данными направлениями превышает $15^{\circ}$, и его отсутствие на дифрактограмме свидетельствует, что разориентация текстурных компонент меньше данного значения.

Проведенные дифрактометрические измерения на длине волны хрома в геометрии $\theta-2 \theta$ также показали, что в пленке есть зерна с ориентированными по поверхности плоскостями $\{110\}$ и $\{001\}$. Учитывая это, можно говорить о многокомпонентной текстуре $\{110\}$, $\{001\}$ и $\{221\}$, которая возможно связана с межзерненными границами. Одним из возможных объяснений текстуры $\{221\}$ является процесс двойникования: для молибдена, как и для других металлов с ОЦК решеткой, плоскостью двойникования является плоскость $\{112\}$, в результате этого направления $\langle 001\rangle$ в исходной и $\langle 221\rangle$ в двойниковой системе оказываются параллельными, а соответствующая текстура $\{221\}$ предположительно является двойником роста текстуры $\{001\}$.

Так как для молибдена нормаль к плоскости с минимальной поверхностной энергией $\{110\}$ не совпадает с направлением быстрого роста [100], то структура образующихся пленок существенно зависит от условий напыления. Если термодинамическое равновесие не успевает установиться, то возможен рост многокомпонентной текстуры. Такой рост при магнетронном напылении молибдена наблюдался неоднократно при различных условиях напыления [15-17], причем текстура $\{001\}$ появлялась при максимальной мощности разряда, и напряжения в этом случае оказывались максимальными. Малый разброс размеров зоны когерентности при измерении под разными углами свидетельствует об отсутствии столбчатой структуры: кристаллиты покрытия имеют примерно одинаковые размеры во всех направлениях. Исходя из этого, размеры области когерентности можно оценить более точно с учетом деформационных напряжений для текстурной компоненты $\{221\}$. Уорреном было показано, что для сферических частиц размера $D$ при двойниковании в ОЦК решетках с плоскостью двойникования $\{211\}$ возможно введение эффективного размера $D_{\mathrm{eff}}^{h k l}$, определяющего ширины дифракционных пиков [18]:

$$
\frac{1}{D_{\mathrm{eff}}^{h k l}}=\frac{1}{D}+\frac{1}{D_{\mathrm{tw}}^{h k l}},
$$

где $D_{\mathrm{tw}}^{h k l}$ - деформационный размер, связанный с уширением из-за микронапряжений и определяемый как параметром решетки и вероятностями двойникования, так и плотностью дефектов упаковки. При этом деформационный размер $D_{\mathrm{tw}}^{h k l}$ зависит от индексов отражения, в частности, отношение размеров для пиков (110) и (211) оказывается равным (табл. 13.3 в [18]):

$$
\frac{D_{\mathrm{tw}}^{110}}{D_{\mathrm{tw}}^{211}}=\sqrt{3} \text {. }
$$

Для определения размера можно использовать только данные по отражениям (110) и (211), поскольку отражение (220) присутствует как в текстуре $\{221\}$, так и $\{001\}$, a (310) относится к текстурной компоненте $\{110\}$. В этом случае из (2) и (3) получаем систему из трех уравнений с тремя неизвестными $\left(D, D_{\mathrm{tw}}^{110}\right.$ и $\left.D_{\mathrm{tw}}^{211}\right)$ :

$$
\left\{\begin{array}{l}
\frac{1}{D_{\mathrm{eff}}^{110}}=\frac{1}{D}+\frac{1}{D_{\mathrm{tw}}^{110}} \\
\frac{1}{D_{\mathrm{eff}}^{211}}=\frac{1}{D}+\frac{1}{D_{\mathrm{tw}}^{211}} \\
D_{\mathrm{tw}}^{110}=\sqrt{3} D_{\mathrm{tw}}^{211}
\end{array}\right.
$$

Решение этой системы уравнений позволяет определить размер сферических частиц:

$$
D=\frac{(\sqrt{3}-1) D_{\mathrm{eff}}^{110} D_{\mathrm{eff}}^{211}}{\sqrt{3} D_{\mathrm{eff}}^{211}-D_{\mathrm{eff}}^{110}}
$$

Подставляя значения $D_{\text {eff }}$ из табл. 1 , получаем величину $D=13.7 \mathrm{~nm}$.

Все это говорит о том, что кинетика роста сама ограничивает размеры частиц по всем направлениям, которые для исследуемого образца покрытия не превышают $14 \mathrm{~nm}$.

\section{Исследование устойчивости покрытия к распылению}

Необходимо отметить, что зеркала оптических диагностик реактора ИТЭР снабжены встроенной системой плазменной очистки. Оценки показывают, что за время эксплуатации зеркала флюенс ионов рабочего газа, используемого для очистки, может на несколько порядков превысить флюенс атомов перезарядки (в основном изотопов водорода). В силу того что в качестве рабочего газа в системах плазменной очистки зеркал не исключено использование $\mathrm{Ar}$, а коэффициент распыления Мо-аргоном в несколько раз больше по сравнению с водородом и гелием, этот газ применялся в качестве рабочего и в наших экспериментах.

Исследование устойчивости покрытия к распылению проводилось посредством измерения спектра отражения

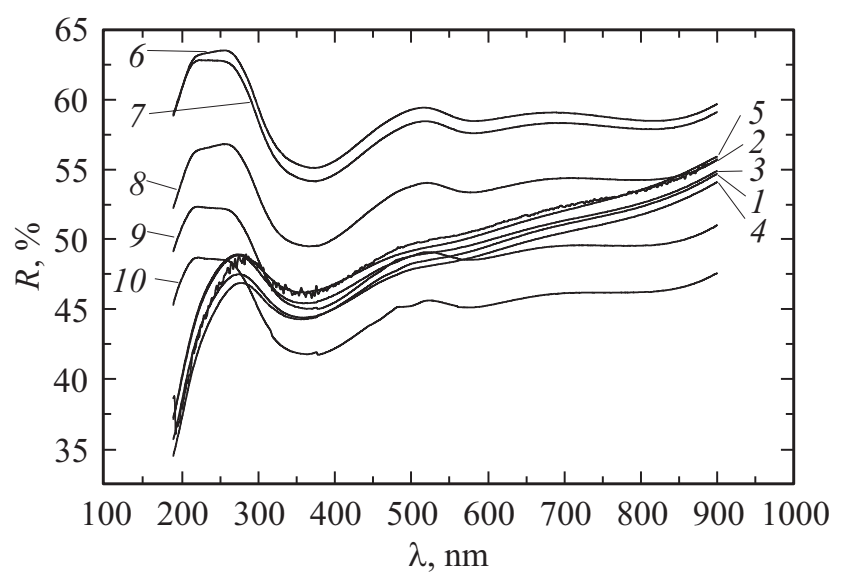

Рис. 3. Зависимости коэффициента зеркального отражения от длины волны излучения при суммарной толщине $h$ распыленного слоя, nm: $1-240,2-460,3-670,4-830,5-990$, $6-1300,7-1470,8-1920,9-2390,10-2870$. 


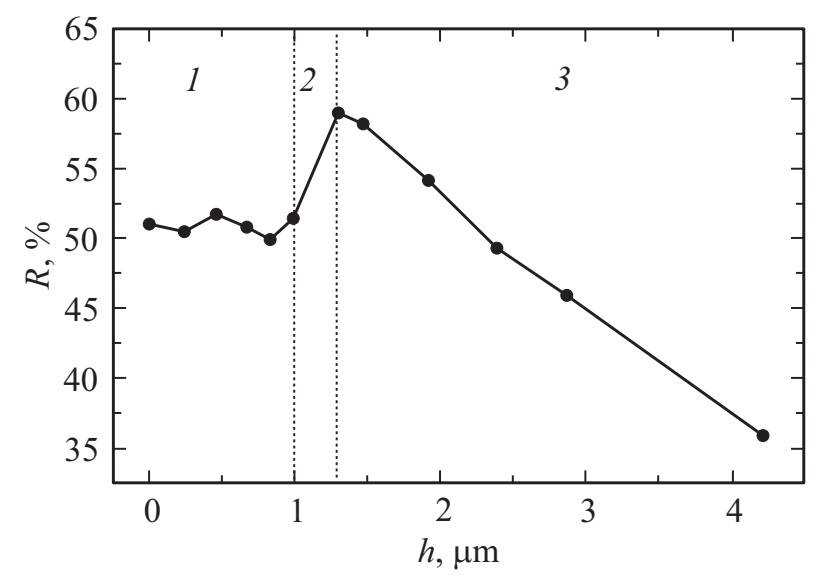

Рис. 4. Зависимость коэффициента зеркального отражения $R$ на длине волны $\lambda=656 \mathrm{~nm}$ от толщины $h$ распыленного слоя, где зона 1 соответствует распылению покрытия, $2-$ распыление поверхностного дефектного слоя подложки, сформированного при полировке, 3 - распыление поликристаллической подложки.

зеркала и на основе данных электронной микроскопии, проведенной после нескольких циклов распыления. При этом использовалась та же конфигурация оборудования, как при финишной очистке подложки перед напылением. Использовался следующий режим распыления: $U=350 \mathrm{~V}, I=200 \mathrm{~mA}, P_{\mathrm{Ar}}=2 \cdot 10^{-2}$ Torr. Плотность тока на зеркало $j=0.7 \mathrm{~mA} / \mathrm{cm}^{2}$. Измерение спектральной зависимости коэффициента зеркального отражения $R$ от длины волны излучения $\lambda$ проводилось на спектрофотометре Perkin Elmer Lambda 850, использовался диагностический луч квадратного сечения $(5 \times 5 \mathrm{~mm})$, угол падения диагностического луча на отражающую поверхность составлял $8^{\circ}$. Полученные зависимости $R(\lambda)$ в диапазоне длин волн $\lambda=(190-900) \mathrm{nm}$ для различных толщин распыленного слоя представлены на рис. 3 .

Отклонение коэффициента зеркального отражения от средней величины при проведении измерения в нескольких точках на поверхности образца не превышало $3 \%$.

Можно выделить две различающиеся формой группы спектральных зависимостей коэффициента отражения. В каждой группе кривые взаимно эквидистантны. Первая группа (кривые $1-5$ на рис. 3 ) соответствует спектральным характеристикам покрытия при разной толщине распыленного слоя. Вторая (кривые 6-10 на рис. 3) распылению поликристаллической подложки после полного удаления покрытия. Поскольку состав покрытия тот же, что и состав поликристаллической подложи, наблюдаемое отличие в спектральных характеристиках можно объяснить только влиянием структуры покрытия на его оптические свойства.

На основании обработки данных, представленных на рис. 3, построена зависимость коэффициента отражения $R$ на длине волны $\lambda=656 \mathrm{~nm}$ (линия $H_{\alpha}$ ) от толщины распыленного слоя $h$, показанная на рис. 4 .

Видно, что при распылении покрытия коэффициент отражения практически не меняется (зона 1 на рис. 4), т. е. покрытие обладает высокой оптической стойкостью к распылению. После полного удаления покрытия первоначальные оптические свойства подложки восстанавливаются (зона 2 на рис. 4). При распылении поликристаллической подложки (зона 3 на рис. 4) падение коэффициента отражения пропорционально толщине распыленного слоя, что согласуется с результатами, представленными в [3]. Значение максимума коэффициента отражения на рис. 4 совпадает с измеренным значением коэффициента отражения подложки перед напылением покрытия, т. е. соответствует полному удалению покрытия с поверхности подложки.
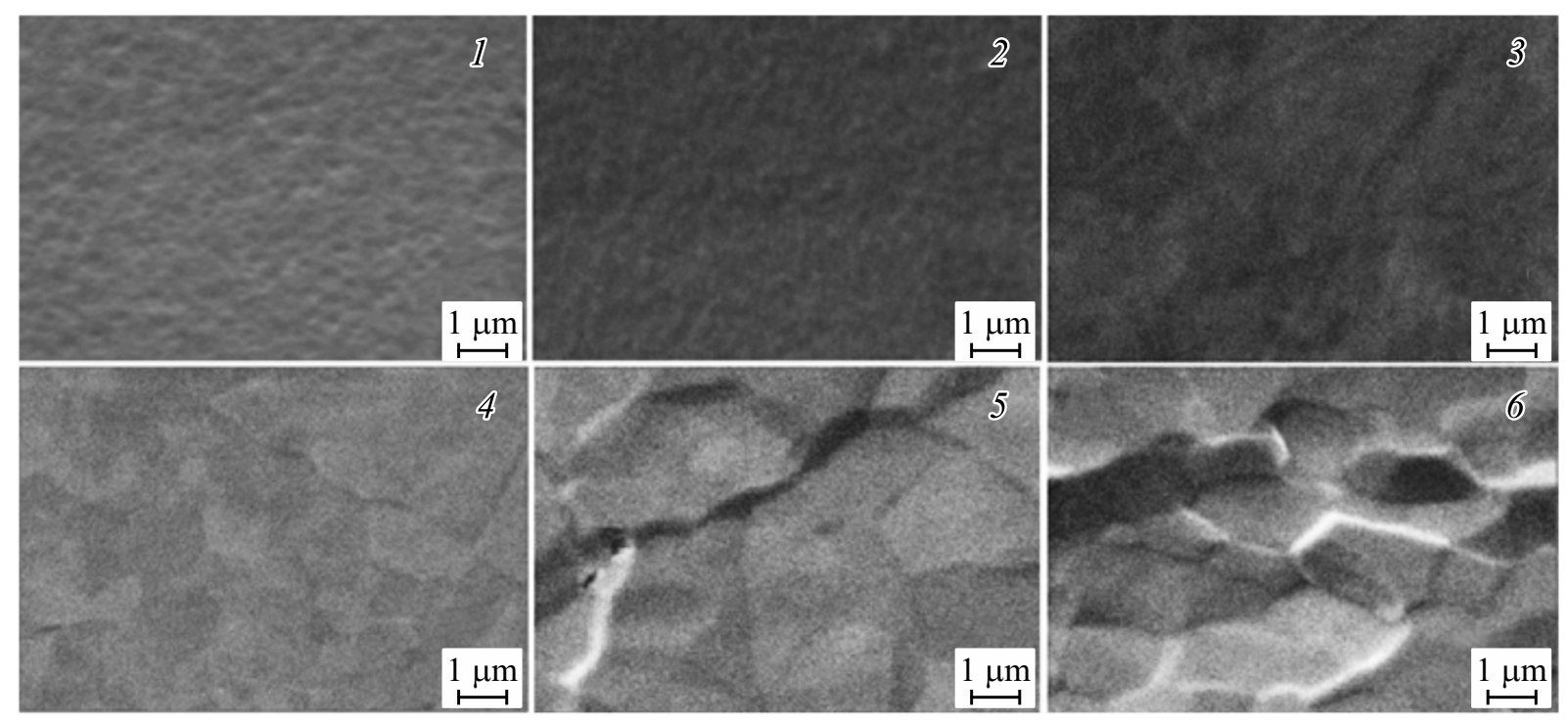

Рис. 5. Изменение морфологии поверхности в зависимости от толщины распыленного слоя $h, \mathrm{~nm}: 1-0,2-990,3-1300$ $\left(R=R_{\max }\right), 4-1470,5-2390,6-4220$. 


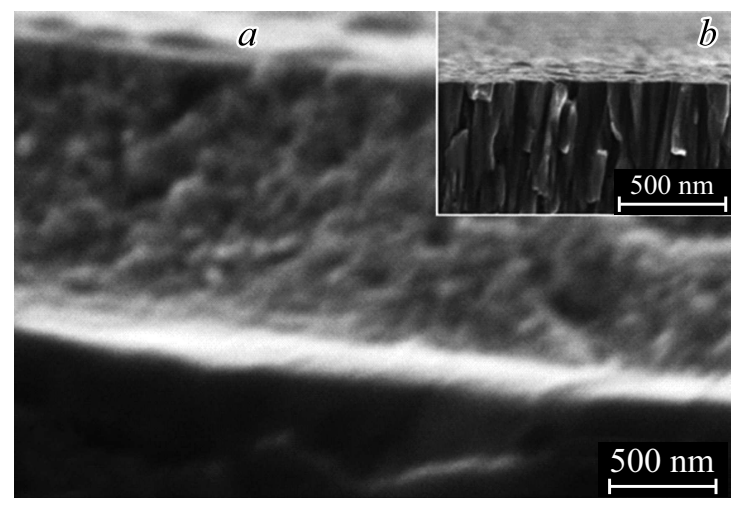

Pис. 6. Структура Мо-покрытия на разломе: магнетронное напыление: $a$ - при наличии, $b-$ без ионного ассистирования в процессе напыления.

Динамика изменения морфологии отражающей поверхности исследовалась с использованием сканирующего электронного микроскопа Carl Zeiss Evo MA10. Результаты представлены на рис. 5.

Из рис. 5 видно, что при распылении покрытия морфология отражающей поверхности практически не меняется. Переход от одной группы спектральных зависимостей коэффициента отражения к другой наблюдается только при полном удалении покрытия. При этом начинают проявляться дефекты полировки подложки в виде царапин (рис. 5,3), не наблюдавшиеся ранее на поверхности покрытия. Это обусловлено обнаруженным ранее эффектом сглаживания микронеровностей при одновременном напылении материала подложки и его распылении [19]. После полного удаления покрытия последующее распыление приводит к развитию рельефа поверхности за счет различия в скорости распыления зерен с разной кристаллографической ориентацией относительно поверхности подложки [2].

Результаты электронной микроскопии разлома покрытия представлены на рис. 6,a. Для сравнения, также показана структура столбчатого Мо-нанокристаллитного покрытия (рис. 6, $b$ ), полученного в сходном режиме магнетронного напыления без дополнительного нагрева подложки и без ионного ассистирования [7].

Морфология поверхности на разломе подтверждает отсутствие столбчатой структуры у покрытия, полученного при магнетронном напылении с одновременным частичным распылением осаждаемого материала.

\section{Теоретический анализ}

\section{Теория роста кристаллитов при напылении, ассистированном распылением}

Рассмотрим процессы, протекающие при одновременном напылении и распылении покрытия, когда скорость осаждения выше скорости травления. При этом термодинамическое равновесие не успевает установиться, и кристаллиты частично растут в направлении максимальной скорости роста (для Мо) $\langle 001\rangle$ с текстурой поверхности $\{001\}$, а не только с текстурой поверхности минимальной энергии $\{110\}$. Для ОЦК кристаллов известно, что плоскостью двойникования может быть плоскость $\{211\}$, в этом случае направление $\langle 001\rangle$ в исходной матрице совпадает с направлением $\langle 221\rangle$ в двойниковой, поэтому из-за двойникования при таком механизме роста возможно образование зерен с текстурой поверхности $\{221\}$, что подтверждается экспериментально.

Покажем, почему при одновременном напылении и распылении образуется мелкозернистая структура покрытия. В работе [19] было показано, что одновременное распыление и напыление материала подложки сглаживает поверхность, причем сглаживание наиболее эффективно, когда скорость распыления и осаждения равны. Это происходит в результате заполнения ям подвижными осаждаемыми атомами и разрушения крупных выступающих объектов распылением. При таком процессе разрушаются также крупные кластеры зародыши, на которых растут кристаллиты, а размер кластера - зародыша ограничен сверху. Действительно, если кластер образуется за счет объединения свободно диффундирующих по поверхности адатомов, то рост числа атомов в кластере $n$ описывается уравнением

$$
\frac{d n}{d t}=\pi D C-\pi j Y_{d} n a^{2},
$$

где $C$ - концентрация свободно диффундирующих по поверхности атомов - адатомов, $D-$ их коэффициент диффузии, $j$ - плотность потока распыляющих ионов, $Y_{d}$ - коэффициент отрыва атома от кластера (оцениваемый как $Y_{d}=2 Y, Y$ - коэффициент распыления), $a-$ межатомное расстояние. Первый член справа в (6) описывает присоединение адатомов к кластеру, второй отрыв атомов от кластера в результате ионного облучения. Заметим, что в двумерной геометрии объединение адатомов и присоединение их к кластеру не зависит от размера кластера. Скорость присоединения адатома к кластеру (или другому адатому) диаметром $d$ равна потоку диффундирующих адатомов $D \operatorname{grad} C$ на кластер с периметром $\pi d$. Принимая градиент концентрации адатомов возле кластера, равным $\operatorname{grad} C=C / d$, получаем, что скорость присоединения адатома к кластеру равна $\pi D C$.

Из уравнения (6) видно, что при размерах кластера больше некоторого значения $n_{\max }$ скорость отрыва атомов от кластера становится больше скорости присоединения адатомов к кластеру. Максимальный размер кластера $n_{\max }$ определяется из (6) при $d n / d t=0$. Тогда

$$
n_{\max }=\frac{D C a^{2}}{j Y_{d}} .
$$

Соответственно размер кластеров и зерен, образующихся из кластеров, равен

$$
d=a n^{1 / 2} .
$$


Концентрация адатомов $C$ описывается уравнением

$$
\frac{d C}{d t}=q-\pi D C^{2}-\pi D N C+j N Y_{S} S
$$

где $q$ - число атомов, появившихся на единице площади в единицу времени. В свою очередь, это число равно

$$
q=q_{0}-j Y,
$$

где $q_{0}-$ скорость осаждения атомов, $j Y-$ скорость распыления, $j$ - плотность потока распыляющих ионов, $Y$ - коэффициент распыления. Второй член справа в (9) отражает объединение адатомов в двухатомные кластеры, третий член - присоединение адатомов к уже существующим кластерам, концентрация которых $N$, последний член описывает отрыв атома от кластера при ионном облучении, причем оторванный атом не распыляется, а остается на поверхности в виде одиночного адатома, $Y_{S}$ - коэффициент отрыва атома от кластера, $S=n a^{2}$ - средняя площадь кластера и его окружения $(n-$ число атомов в кластере, $a$ - межатомное расстояние), при попадании в которую атом выбивается из кластера. Энергия, необходимая для отделения атома от кластера, равна

$$
E_{d}=\frac{H}{3},
$$

где $H$ - энергия сублимации [20]. Принимая во внимание, что

$$
\begin{gathered}
Y \sim 1 / H, \\
Y_{S} \sim\left(1 / E_{d}-1 / H\right),
\end{gathered}
$$

в результате получаем

$$
Y_{S} \approx 2 Y \text {. }
$$

В данном случае мы пренебрегаем распадом кластера в результате теплового возбуждения, которое существенно только при высоких температурах.

При малых временах, когда концентрация кластеров мала, последние два члена в (9) малы, и при времени облучения

$$
t_{S}>(\pi q D)^{-1 / 2}
$$

концентрации адатомов равна

$$
C=\left(\frac{q}{\pi D}\right)^{1 / 2}
$$

Максимальное число атомов в кластере при этом

$$
n_{\max }=\left(\frac{q D}{\pi}\right)^{1 / 2}\left(a^{2} j Y\right)^{-1} .
$$

Оценки, сделанные на основе величины коэффициента диффузии $D \approx 10^{-9} \mathrm{~cm}^{2} / \mathrm{s}$ [19] для нашего случая, дают величину $n_{\max } \approx 10 \mathrm{~nm}$. Отсюда следует, что существовавшие до напыления кластеры с числом атомов, больше $n_{\max }$, разрушаются ионной бомбардировкой. Таким образом, ионная бомбардировка не позволяет образовываться в пленке крупным кристаллитам и способствует уменьшению разброса (дисперсии) их размеров.

Число кластеров растет, и при большой концентрации кластеров расстояние между ними

$$
\Delta_{c l}=\left(N^{-1 / 2}-a n_{\min }^{1 / 2}\right)
$$

становится меньше, чем расстояние между адатомами

$$
\Delta_{a a}=C^{-1 / 2} \text {. }
$$

Тогда образование новых кластеров прекращается, и все появившиеся адатомы присоединяются к уже имеющимся кластерам. При этом размер кластеров, на которых растут зерна покрытия, равен

$$
\begin{aligned}
d & =N^{-1 / 2}=C^{-1 / 2}+a n^{1 / 2} \\
& =D^{1 / 4}\left[\left(\frac{\pi}{q}\right)^{1 / 4}+\left(\frac{q}{\pi}\right)^{1 / 4}\left(j Y_{S}\right)^{-1 / 2}\right] .
\end{aligned}
$$

Отсюда видно, что размер зерен покрытия имеет минимум при $q=\pi j Y_{S}$, т. е. при скорости осаждения

$$
q_{0}=j\left(Y+\pi Y_{S}\right)
$$

а минимальный размер зерен покрытия равен

$$
d=2\left(\frac{D}{j Y_{S}}\right)^{1 / 4}
$$

Оценка коэффициента диффузии адатомов по поверхности молибдена была сделана в [19]. Разброс значений $D$ для разных граней кристалла достаточно велик, но для температуры мишени $T<500 \mathrm{~K}$ значения $D<10^{-9} \mathrm{~cm}^{2} / \mathrm{s}$.

В нашем эксперименте скорость осаждения $q$ оценивалась как $q=h N / T(h-$ толщина покрытия, $N$ число атомов в единице объема покрытия, $T-$ время напыления), а скорость распыления $j Y$ пересчитывалась из эксперимента по очистке поверхности с учетом зависимости от энергии ионов. Такие оценки дают $q=3.3 \cdot 10^{15} \mathrm{~cm}^{-2} \mathrm{~s}^{-1}, j Y=7.3 \cdot 10^{16} \mathrm{~cm}^{-2} \mathrm{~s}^{-1}$, и при $D=10^{-9} \mathrm{~cm}^{2} / \mathrm{s}$ размер кластера, на котором растут зерна, составляет $d \approx 12 \mathrm{~nm}$. Размер кластера, являющегося ядром для растущего зерна, определяет конечный поперечный размер кристаллита. Кристаллиты растут эпитаксиально на затравочном кластере и при напылении без распыления образуют текстурированную столбчатую структуру $[7,8]$.

Рассмотрим теперь эпитаксиальный рост кристаллитов на уже образовавшихся кластерах. При одновременном осаждении материала и распылении рост кристаллов имеет особенности. Распыление различных граней не одинаково: меньше всего распыляются наиболее „прозрачные“ низкоиндексные грани. Коэффициент распыления низкоиндексных граней на (20-30)\% меньше [21], чем коэффициент распыления поликристалла. Поэтому 
соотношение скоростей осаждения и распыления для них сдвинуто относительно среднего значения в сторону увеличения скорости осаждения. Таким образом, низкоиндексные грани, помимо фактора энергетической „выгодности“, при одновременном распылении имеют дополнительный стимул расти быстрее, чем высокоиндексные грани. Однако для мелких кристаллитов, растущих в окружении других кристаллитов, картина может быть обратной. На низкоиндексных гранях скорость осаждения атомов сильнее преобладает над скоростью распыления, т.е. скорость появления атомов $q$ на поверхности низкоиндексных граней больше. Разность скоростей появления атомов на разных гранях определяется разностью их коэффициентов распыления $Y$ и равна

$$
\Delta q=-j \Delta Y .
$$

Концентрация $C$ адатомов на поверхности растущего кристалла определяется, во-первых, закреплением адатома у поверхности ступеньки, а во-вторых, диффузией на соседние зерна, где $q$ отличается от $q$ данного зерна. В установившемся режиме концентрация адатомов на зерне круглого сечения описывается уравнением

$$
q+\frac{D}{r} \frac{\partial}{\partial r}\left(r \frac{\partial C}{\partial r}\right)-\frac{C}{\tau}=0,
$$

где $\tau$ - время, за которое адатом закрепится у ступеньки на поверхности растущего кристалла, равное времени диффузии адатома до ступеньки на поверхности, к которой присоединяется атом

$$
\tau \approx \frac{l^{2}}{D}
$$

где $l$ - среднее расстояние между ступеньками (в дальнейшем используется $l \ll R)$. Условием на границе зерна $r=R$ является заданная концентрация $C(R)$, которую для простоты, полагая окружение данного зерна симметричным, положим равной

$$
C(R)=\frac{\Delta q \tau}{2}=\frac{j \Delta Y \tau}{2} .
$$

В нулевом приближении можно пренебречь диффузией, тогда $C=q \tau$. Следующим приближением, конечным при $r=0$ и удовлетворяющим граничному условию (14), является

$$
\begin{gathered}
C=C_{0}-A r^{2}, \\
C_{0}=q \tau, \\
A=\frac{j \tau \Delta Y}{2 R^{2}} .
\end{gathered}
$$

Скорость вертикального роста кристаллита равна

$$
\frac{d h}{d t}=\frac{C a^{3}}{\tau}
$$

и в силу зависимости концентрации адатомов $C$ от расстояния до центра кристаллита также зависит от $r$.
Таким образом, через время $t$ поверхность кристаллита приобретает наклон

$$
\frac{d h}{d r}=-2 A r a^{3} t=-\frac{j \Delta Y \tau R^{2} t}{r a^{3}} .
$$

Рост кристаллитов на наклонной поверхности происходит эпитаксиально уже на другой кристаллографической плоскости, т.е. растет кристаллит другой ориентации, наиболее вероятно - двойниковый. Критерием начала роста кристаллита другой ориентации можно считать равенство

$$
\left|\frac{d h}{d r}\right|=1
$$

при условии, что

$$
r=R / 2,
$$

что достигается за время, равное

$$
t=\frac{2 R}{a^{3} j \Delta Y} .
$$

За это время центральная часть кристаллита вырастает на высоту

$$
h=q a^{3} t=\frac{2 R q}{j \Delta Y} .
$$

Заметим, что если разность коэффициентов распыления велика и выполняется условие

$$
\left|\frac{q}{j \Delta Y}\right| \ll 1,
$$

то высота кристаллита мала, т.е. $h \ll R$. Это можно интерпретировать как малое представительство кристаллитов с гранями, ориентированными параллельно поверхности с малым коэффициентом распыления. Если же разность коэффициентов мала и

$$
\left|\frac{q}{j \Delta Y}\right| \gg 1,
$$

то такие зерна растут как единое целое с одинаковой скоростью. Если такая группа зерен имеет $q$ меньше, чем окружающие зерна, то она растет медленнее, и окружающие мелкие зерна обгоняют ее и перекрывают сверху. Если группа зерен имеет $q$ больше, чем окружающие зерна, то увеличивается поток диффундирующих адатомов на окружающие мелкие зерна, что ограничивает высоту этой группы атомов. Если быстрорастущая группа зерен имеет радиус $R$, окружающие зерна радиус $R_{1}<R$, а соответственно скорости появления атомов на поверхности $q$ и $q_{1}<q$, то граничное условие $(26)$ для концентрации адатомов на поверхности быстрорастущей группы следует заменить на

$$
\left.\frac{d C}{d r}\right|_{R} \approx \frac{\Delta C}{R_{1}}=2 A R=\frac{j \Delta Y \tau}{R_{1}} .
$$

Отсюда следует, что предельная высота этой группы зерен равна

$$
h=\frac{2 R_{1} q}{j \Delta Y},
$$


т.е. такая же, как высота у окружающих мелких зерен. Так что в основном представлены кристаллиты высотой примерно равной среднему диаметру зерен

$$
h=2 R \text {. }
$$

Следует отметить, что коэффициент распыления $Y$ возрастает при увеличении угла наклона $\theta$ поверхности кристаллита. Однако при размерах неоднородности меньше дебаевского слоя плазмы поток облучающих ионов нормален к средней плоскости поверхности, а плотность потока ионов на локальную поверхность $j \cos (\theta)$, так что зависимость $j Y$ от $\theta$ слабая и не меняет описанного процесса. В любом случае представлены кристаллиты, у которых $q /(j \Delta Y) \approx 1$.

Таким образом, при напылении с одновременным распылением формируется мелкозернистая структура покрытия с зернами размером $\sim 10 \mathrm{~nm}$. Это значение близко к размеру кристаллитов, полученному из анализа дифрактограммы покрытия.

\section{Оценка размера кристаллитов из анализа спектра отражения покрытия}

Рассмотрим влияние размеров кристаллитов в покрытии на его оптические свойства применительно к мелкодисперсным покрытиям. Формула Френеля позволяет выразить коэффициент отражения $R$ при нормальном падении излучения из воздуха (вакуума) на отражающую поверхность через диэлектрическую проницаемость формулой [22]

$$
\begin{aligned}
& R=\frac{(u-1)+k^{2}}{(u+1)^{2}+k^{2}}, \\
& u=\sqrt{\frac{|\varepsilon|+\operatorname{Re}(\varepsilon)}{2}}, \\
& k=\sqrt{\frac{|\varepsilon|-\operatorname{Re}(\varepsilon)}{2}},
\end{aligned}
$$

где $R-$ коэффициент отражения, $\varepsilon-$ комплексная диэлектрическая проницаемость материала, $u, k-$ действительная (показатель преломления) и мнимая (коэффициент экстинкции) части комплексного показателя преломления.

Диэлектрическая проницаемость металла содержит вклад как электронов проводимости (друдевская составляющая), так и несвободных электронов (лоренцевская составляющая, учитывающая межзонные переходы). Изза дополнительного рассеяния электронов проводимости на границах нанокристаллитов, она может быть выражена следующим образом:

$$
\begin{aligned}
\varepsilon_{\text {corr }}(\omega)=\varepsilon_{\text {mono }}(\omega) & -\varepsilon_{\text {Dr-mono }}\left(\omega, \gamma_{\text {mono }}\right) \\
& +\varepsilon_{\text {Dr-nano }}\left(\omega, \gamma_{\text {nano }}\right),
\end{aligned}
$$

где $\varepsilon_{\mathrm{corr}}(\omega)$ - диэлектрическая проницаемость нанокристаллического Мo, $\varepsilon_{\text {mono }}(\omega)$ - диэлектрическая про-

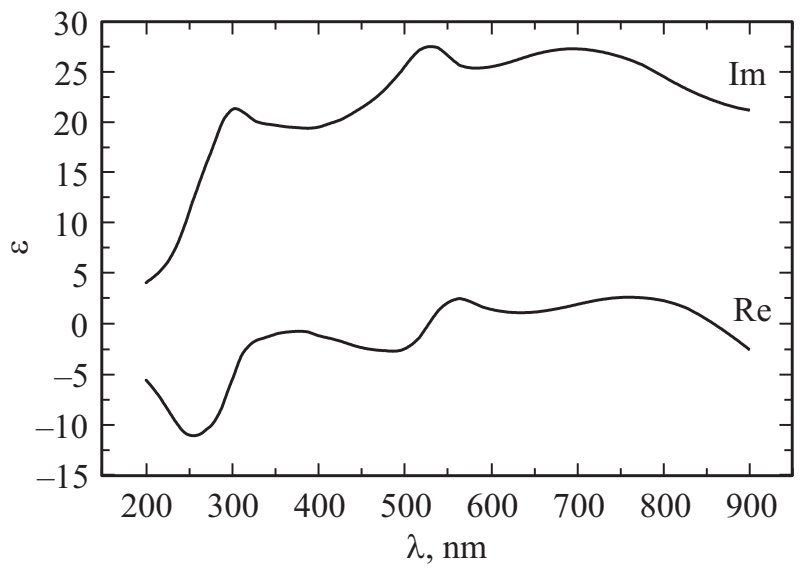

Рис. 7. Действительная Re и мнимая Im части диэлектрической проницаемости молибдена [23].

ницаемость монокристаллического Мо, $\varepsilon_{\mathrm{Dr}}$ - друдевская составляющая.

$$
\begin{gathered}
\gamma_{\text {mono }}=1 / \tau_{\text {mono }}, \\
\gamma_{\text {nano }}=1 / \tau_{\text {nano }},
\end{gathered}
$$

где $\tau_{\text {mono }}-$ среднее время релаксации электрона в монокристаллическом Mo, $\tau_{\text {nano }}$ - среднее время релаксации электрона в Мо-нанокристаллическом покрытии.

Друдевская составляющая зависит от частоты падающего излучения $\omega$ и двух параметров - оптической плазменной частоты $\omega_{\text {opt }}$ и обратного времени релаксации $\gamma$ - и имеет вид [23]

$$
\varepsilon_{\mathrm{Dr}-i}\left(\omega, \omega_{\mathrm{opt}}, \gamma_{i}\right)=1-\frac{\omega_{\mathrm{opt}}^{2}}{\omega^{2}+\gamma_{i}^{2}}+\frac{i \gamma_{i} \omega_{\mathrm{opt}}^{2}}{\left(\omega^{2}+\gamma_{i}^{2}\right) \omega}
$$

где индекс $i$ указываюет на тип образца (mono или nano). Для Мо $\omega_{\text {opt }}=5.4 \cdot 10^{16} \mathrm{rad} / \mathrm{s}$ [24]. В оптическом диапазоне действительная и мнимая части диэлектрической проницаемости молибдена представлены на рис. $7[25,26]$.

В мелкозернистом кристалле появляется дополнительный масштаб времени $\left(\tau_{\text {eff }}\right)$ - отношение размера зерна $(D)$ к скорости электрона на уровне Ферми $\left(V_{f}\right)$

$$
\tau_{\mathrm{eff}}=\frac{D_{\mathrm{opt}}}{V_{f}} .
$$

При этом выполняется условие

$$
\tau_{\text {nano }}=\frac{\tau_{\text {eff }} \tau_{\text {mono }}}{\tau_{\text {eff }}+\tau_{\text {mono }}} .
$$

Для Мо скорость электрона на уровне Ферми $V_{f}=$ $=1.7 \cdot 10^{6} \mathrm{~m} / \mathrm{s}, \tau_{\text {mono }}=1.2 \cdot 10^{-14} \mathrm{~s}[24]$. Уравнения (48), (49) позволяют определить размер зерна $D$ :

$$
D_{\mathrm{opt}}=\frac{\tau_{\mathrm{mono}} \tau_{\text {nano }} V_{f}}{\tau_{\text {mono }}-\tau_{\text {nano }}} .
$$



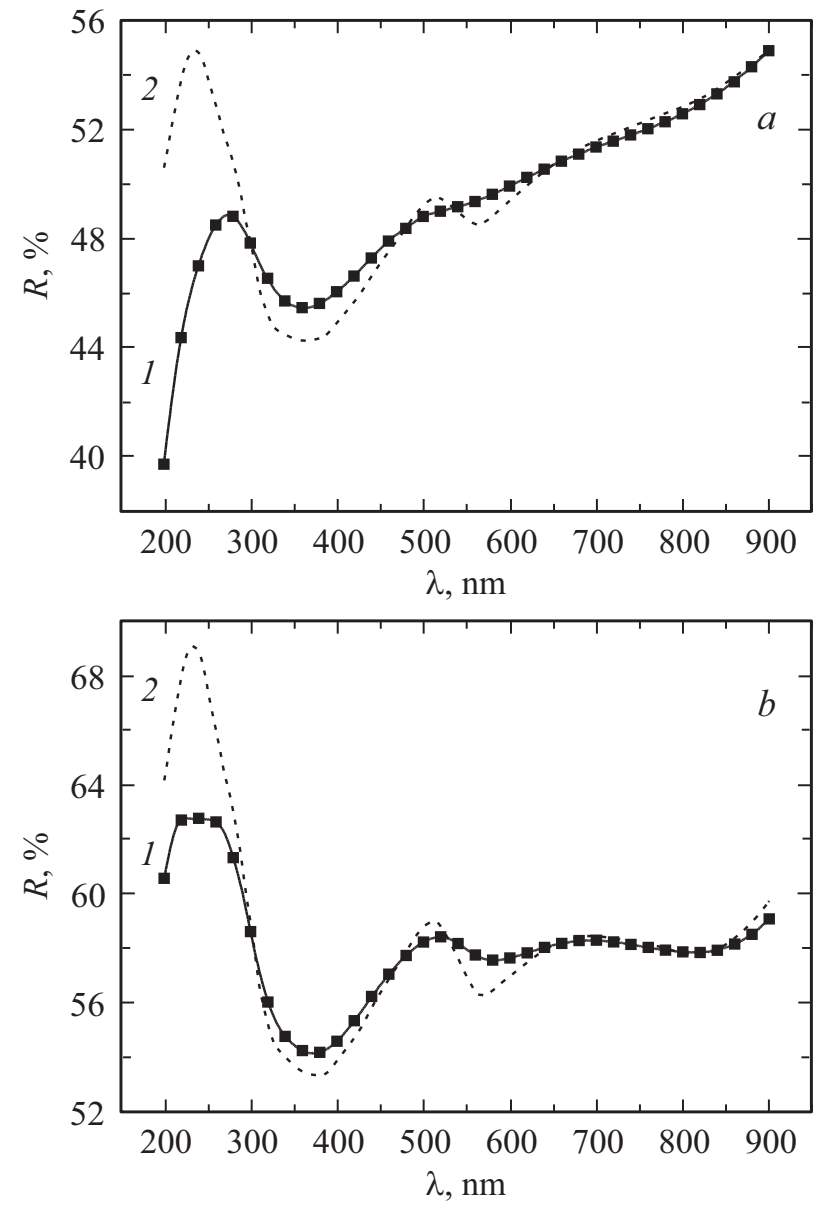

Рис. 8. Измеренные и расчетные значения $R(\lambda)$ для нанокристаллитного покрытия $(a)$ и поликристаллической подложки $(b): 1$ - эксперимент, 2 - расчет.

С учетом вышесказанного выражение (41) можно записать в виде

$$
R\left(\varepsilon_{\text {corr }}\right)=A\left(1-2 \frac{\sqrt{2}\left|\varepsilon_{\text {corr }}\right| \sqrt{\left|\varepsilon_{\text {corr }}\right|+\operatorname{Re}\left(\varepsilon_{\text {corr }}\right)}+\sqrt{2} \times}{\times \sqrt{\left|\varepsilon_{\text {corr }}\right|+\operatorname{Re}\left(\varepsilon_{\text {corr }}\right)}-2 \varepsilon_{\text {corr }}-2 \operatorname{Re}\left(\varepsilon_{\text {corr }}\right)}\right),
$$

где $A-$ подгоночный коэффициент (зависит от шероховатости поверхности и других факторов, определяющих диффузное рассеяние).

Среднее время релаксации электрона $\tau_{\text {nano }}$ в Мoнанокристаллическом покрытии и поликристаллической подложке и коэффициент $A$ при расчете подбирались по критерию наименышего отклонения между расчетной и экспериментальной зависимостями $R(\lambda)$ в диапазоне длин волн (500-900) nm. В этой зоне в меньшей степени проявляется влияние шероховатости и остаточных загрязнений на поверхности зеркала. Анализ экспериментально измеренных спектральных зависимостей для исследуемого нанокристаллического Мо-покрытия и поликристаллической подложки после распыления покрытия проводился с использованием программы Mathcad 14.
Таблица 2. Параметры для расчета среднего размера зерен

\begin{tabular}{l|c|c|c}
\hline \multicolumn{1}{c|}{ Тип Мо-зеркала } & $A$ & $\tau_{\text {nano }}, \mathrm{s}$ & $D_{\text {opt }}, \mathrm{nm}$ \\
\hline С нанокристаллическим покрытием & 98 & $9.0 \cdot 10^{-15}$ & 13 \\
Поликристаллическое & 79 & $4.6 \cdot 10^{-15}$ & 61
\end{tabular}

Экспериментальные зависимости $R(\lambda)$ для нанокристаллического покрытия и поликристаллической подложки после его полного удаления (зависимости 3 и 7 на рис. 3) и соответствующие результаты расчета показаны на рис. 8 .

Подгоночные значения $\tau_{\text {nano для нанокристаллическо- }}$ го покрытия и поликристаллической подложки использовались для расчета $D_{\text {орt}}$. Использованные параметры и результаты расчета размера зерен представлены в табл. 2.

Обозначим $D_{\text {орt, }}$ полученные для нанокристаллического покрытия и поликристалла, соответственно как

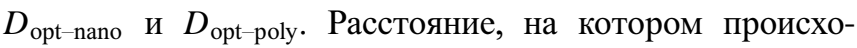
дит релаксация электрона только за счет рассеяния на фононах для Мо, составляет $D_{f}=V_{f} \tau_{\text {mono }} \approx 20 \mathrm{~nm}$. Зерна в Мо-поликристалле имеют размеры порядка $1 \mu \mathrm{m}$ (рис. 5), т.е. величину примерно в 50 раз больше, чем $D_{f}$. Следовательно, для поликристаллического молибдена влияние границ зерен на оптические свойства проявляется слабо, и время жизни электрона проводимости определяется в основном рассеянием на фононах. При этом полученное значение $D_{\text {poly можно интерпрети- }}$ ровать как оценку значения $D_{f}$, полученного из анализа оптических измерений.

Для нанокристаллитного покрытия, поскольку $D_{\text {opt-nano }}<D_{f}$, основное рассеяние происходит на границах кристаллитов, т.е. $D_{\text {opt-nano }}$ соответствует среднему размеру кристаллитов в нанокристаллическом покрытии. На основании полученных результатов можно сделать вывод, что оптические свойства нанокристаллических материалов в основном определяются их мелкодисперсной структурой.

\section{Заключение}

На основании полученных экспериментальных результатов можно сделать заключение, что Мо-покрытия, полученные при магнетронном напылении с одновременным низкоэнергетическим ионным распылением, имеют нанокристаллическую структуру с высокой степенью однородности, обладают многокомпонентной текстурой $\{001\},\{110\}$ и $\{221\}$ и состоят из кристаллитов с характерным размером около $10 \mathrm{~nm}$. Этим обеспечивается высокая стойкость к распылению. Разработана теоретическая модель формирования кристаллитов покрытия в рассматриваемых условиях. Выявлено, что спектральный коэффициент зеркального отражения покрытия в диапазоне $\lambda=(200-900) \mathrm{nm}$ значительно 
отличается от поликристаллического молибдена. Разработана методика оценки размера кристаллитов в нанокристаллическом покрытии на основе анализа его спектрального коэффициента зеркального отражения. Все три метода оценки размеров кристаллитов (из рентгеновских измерений, на основе анализа спектрального коэффициента зеркального отражения и теоретического расчета) дали близкую величину характерного размера кристаллитов в покрытии. Предложенная оригинальная методика формирования покрытий позволяет в широких пределах варьировать размер кристаллитов в покрытии посредством выбора соотношения скорости осаждения и распыления, а также температуры подложки при напылении. Можно предположить, что подбор оптимального режима напыления и температуры подложки позволит обеспечить высокую стойкость к распылению без существенного изменения спектральной характеристики для используемого материала. Обнаруженный эффект влияния размеров нанокристаллитов на оптические свойства покрытия представляет самостоятельный интерес и может быть использован для экспресс-анализа наличия нанокристаллической структуры покрытия, исследования процессов его старения или структурной модификации при внешних воздействиях.

\section{Список литературы}

[1] Orlinski D.V., Voitsenya V.S., Vukolov K.Yu. // Plasma Devices and Operations. 2007. Vol. 15. N 1. P. 33-75.

[2] Miyamoto M., Takaoka H., Ono K. et al. // J. Nucl. Mater. 2014. Vol. 455. P. 297-300.

[3] Voitsenya V.S., Bardomid A.F., Belyaeva A.I. et al. // Nukleonika. 2012. Vol. 57. N 2. P. 157-162.

[4] Orlovskiy I., Alekseev A., Andreenko E. et al. // $29^{\text {th }}$ Symp. on Fusion Technol. Prague. 2016.

[5] Orlovskiy I., Alekseev A., Andreenko E. et al. // Fusion Engineer. Design. 2015. Vol. 96-97. P. 899-902.

[6] Bardomid A.F., Belyaeva A.I., Bondarenko V.N. et al. // Phys. Scr. 2006. Vol. 123. P. 89-93.

[7] Рогов А.В., Вуколов К.Ю. // ЖТФ. 2006. т. 76. Вып. 4. C. $109-112$.

[8] Eren B., Marot L., Litnovsky A. et al. // Fusion Engineer. Design. 2011. N 86. P. 2593-2596.

[9] Рогов А.В., Вуколов К.Ю., Горшков Ф.В., Гуреев В.М. // ВАНТ. Серия: Термоядерный синтез, 2005. № 2. С. 39-54.

[10] Рогов А.В., Капустин Ю.В. // Успехи прикладной физики. 2016. Т. 4. № 3. С. 240-246.

[11] Носкова Н.И., Мулюков Р.Р. Субмикрокристаллические и нанокристаллические металлы и сплавы. Екатеринбург: УpO PAH, 2003. $279 \mathrm{c}$.

[12] Рогов А.В., Лозован А.А. // Тр. XVIII Междунар. конф. „Взаимодействие ионов с поверхностью ВИП-2007“. Москва. 2007. Т. 3. С. 90-93.

[13] Ranjan M., Kalathiparambil K.K., Vaghela N.P., Mukherjee S. // Plasma Processes and Polymers. 2007. N 7. P. $1030-1035$.

[14] Hindmarch A.T., Parkes D.E., Rushforth A.W. // Vacuum. 2012. Vol. 86. P. 1600-1604.
[15] Krishnan R., Riley M., Lee S., Lu T.-M. // Thin Sol. Films. 2011. Vol. 519. P. 5429-5432.

[16] $\mathrm{Li}$ W., Yan X., Aberle A.G., Venkataraj S. // Proc. Engineer. 2016. Vol. 139. P. 1-6.

[17] Sheu H.-H., Yang C.-C., Liang S.-C. et al. // J. C.C.I.T. 2014. Vol. 43. N 2. P. 61-68.

[18] Warren B.E. X-ray diffraction. NY.: Addison Wesley, 1968. P. 311,312 .

[19] Рогов А.В., Нагель М.Ю., Мартыненко Ю.В. // ВАНТ. Серия: Термоядерный синтез. 2013. Т. 36. № 2. С. 19-24.

[20] Кнаке О., Странский И.Н. // УФН. 1959. Т. 68. С. 261.

[21] Распыление твердых тел ионной бомбардировкой / Под ред. Р. Бериш. Вып. 1. Физическое распыление одноэлементных твердых тел. М.: Мир, 1984. 336 с.

[22] Гуревич М.М. Введение в фотометрию. Л.: Энергия, 1968. $244 \mathrm{c}$.

[23] Вартанян Т.А., Ващенко Е.В. Введение в наноплазмонику. Уч. пособие. СПб.: НИУИТМО, 2012. 86 с.

[24] Кириллова М.М., Номерованная Л.В., Носков М.М. // ЖЭТФ. 1971. Т. 60. № 6. С. 2252-2259.

[25] Marsillac S., Barreau N., Khatri H. et al. // Phys. Stat. Sol. C. 2008. Vol. 5. N 5. P. 1244-1248.

[26] Электронный ресурс. Режим доступа: http://www.filmetrics.com/refractive-indexdatabase/Mo/Molybdenum. 